\title{
Corrigendum: eC-CLEM: flexible multidimensional registration software for correlative microscopies
}

Perrine Paul-Gilloteaux, Xavier Heiligenstein, Martin Belle, Marie-Charlotte Domart, Banafshe Larijani, Lucy Collinson, Graça Raposo \& Jean Salamero

Nat. Methods 14, 101-103 (2017); doi:10.1038/nmeth.4170; corrected after print 10 February 2017

In the version of this piece initially published, Xavier Heiligenstein, Graça Raposo, and Jean Salamero were affiliated with Structure Fédérative de Recherche François Bonamy, INSERM, CNRS, Université de Nantes, Nantes, France in error. These affiliations have been removed. The grant number ANR-10-INSB-04 was incorrect; it has been replaced with the correct grant number, ANR-10-INBS-04. The errors have been corrected in the HTML and PDF versions of the piece as of 10 February 2017.

\section{Erratum: Faster brain imaging}

\author{
Nina Vogt \\ Nat. Methods 14, 34 (2017); doi:10.1038/nmeth.4118; corrected after print 10 February 2017 \\ In the version of this piece initially published, the page numbers of an article were incorrectly cited. The original citation read Neuron $\mathbf{9 2}$, \\ 1-16, 2016; it has been updated to read Neuron 92, 723-738. The error has been corrected in the HTML and PDF versions of this piece as \\ of 10 February 2017.

\section{Erratum: Epitranscriptome sequencing technologies: decoding RNA modifications}

\author{
Xiaoyu Li, Xushen Xiong \& Chengqi Yi \\ Nat. Methods 14, 23-31 (2017); published online 29 December 2017; corrected after print 10 February 2017 \\ In the version of this article initially published, author affiliation numbers were incorrect. Xiaoyu Li originally had affiliation 1; this \\ has been changed to affiliations 1 and 2. Xushen Xiong originally had affiliations 1 and 2; these have been changed to affiliations 1-3. \\ Chengqi Yi originally had affiliations 1 and 3; these have been changed to affiliations 2 and 4 . The error has been corrected in the HTML \\ and PDF versions of the article.
}

